\title{
Cinética da proteína total, fibrinogênio e ceruloplasmina em cordeiros Santa Inês durante os primeiros cinco meses de vida
}

\section{Kinetics of total protein, fibrinogen, and ceruloplasmin in Santa Inês lambs during the first five months of life}

\author{
Jean Silva Ramos, ${ }^{* *}$ Aloisio Bitencourt Nascimento, ${ }^{* *}$ José Tadeu Raynal Rocha Filho, ${ }^{* *}$ Maria Consuêlo Caribé Ayres, ${ }^{* \star}$ \\ Alberto Lopes Gusmão, ${ }^{* *}$ Bruno Lopes Bastos, ${ }^{* * *}$ José Eugênio Guimarães ${ }^{* *}$
}

\begin{abstract}
Resumo
Objetivou-se estudar a cinética da proteína total, fibrinogênio e ceruloplasmina durante os primeiros cinco meses de vida, em cordeiros saudáveis da raça Santa Inês, no município de São Gonçalo dos Campos, Bahia, Brasil. Amostras de sangue foram colhidas de 22 animais, ao longo de onze momentos: logo após o parto (T0), 12 (T1), 24 (T2), 48 horas (T3), sete (T4), 15 (T5), 30 (T6), 60 (T7), 90 (T8), 120 (T9) e 150 dias de vida (T10). A proteína total e o fibrinogênio plasmáticos foram analisados por meio de refratômetro clínico e pela técnica de desnaturação pelo calor, respectivamente, enquanto que a determinação da ceruloplasmina sérica se baseou em sua atividade oxidásica. Para análise estatística utilizou-se o programa SPSS versão 18; os dados com distribuição não paramétrica foram submetidos ao teste de Friedman para avaliar o efeito do tempo, enquanto que as comparações múltiplas pelo teste de Wilcoxon permitiram a identificação das diferenças entre os momentos, adotando-se nível de significância de $5 \%(p<0,05)$. A proteína total apresentou o menor valor no T0 diferindo estatisticamente dos demais tempos, com pico às 12 horas (T1), porém estabilizando-se até o final do experimento. O fibrinogênio não apresentou diferença estatística entre os tempos. De T1 (12h) a T3 (48h) constatou-se baixos valores de ceruloplasmina, muito embora às 24 horas (T2) tenha diferido estatisticamente $(p<0,05)$, em relação ao T0. A partir do sétimo dia (T4) a concentração desta proteína aumentou significativamente, atingindo pico nos tempos T8 (90 dias) e T9 (120 dias). Foi possível estabelecer a cinética das proteínas estudadas, identificar os principais momentos com alterações e sugerir os fatores associados com as mudanças observadas.
\end{abstract}

Palavras-chave: neonatos, proteínas de fase aguda, resposta de fase aguda.

\begin{abstract}
The objective was to study the kinetics of the total protein, fibrinogen, and ceruloplasmin during the first five months of life in healthy lambs Santa Inês, in São Gonçalo dos Campos, Bahia, Brazil. Blood samples were collected from 22 animals, over eleven times: immediately after birth (T0), 12 (T1), 24 (T2), 48 hours (T3), seven (T4), 15 (T5), 30 (T6), 60 (T7), 90 (T8) 120 (T9), and 150 days of age (T10). The total protein and plasma fibrinogen were analyzed by means of a clinical refractometer and the heat denaturation technique, respectively, while the determination of serum ceruloplasmin was based on its oxidase activity. For statistical analysis, the SPSS version 18 program was used; the non-parametric data were submitted to the Friedman test to evaluate the effect of time, whereas the multiple comparisons by the Wilcoxon test allowed the identification of the differences between the moments, adopting a significance level of $5 \%(P<0.05)$. The total protein presented the lowest value at T0 differing statistically from the other times, with a peak at 12 hours (T1), but stabilizing until the end of the experiment. Fibrinogen is not able to differentiate between the times. Between T1 (12h) and T3 (48h), low values of ceruloplasmin were observed, although at 24 hours (T2) it differed statistically $(p<0.05)$ in relation to T0. On the seventh day (T4) the concentration of this protein increased significantly, reaching a peak at T8 (90 days) and T9 (120 days). It was possible to establish the kinetics of the proteins studied, to identify the main moments with alterations and to suggest the factors associated with the observed changes.
\end{abstract}

Keywords: neonates, acute phase proteins, acute phase response.

\section{Introdução}

A ovinocultura tem um mercado promissor em que se observa um aumento do consumo e produção da carne ovina nos últimos anos, especialmente na região Nordeste (Viana, 2008). Com o crescimento desse mercado faz-se necessária a utilização de novos marcadores biológicos que contribuam na detecção dos processos patológicos. Neste contexto, as proteínas de fase aguda (PFA) vêm sendo foco de estudos, em associação com a clínica médica, uma vez que estão relacionadas às reações sistêmicas frente a estímulos inflamatórios, infecciosos, traumáticos e neoplásicos (Eckersall e Bell, 2010).

\footnotetext{
${ }^{*}$ Recebido em 29 de julho de 2015 e aceito em 10 de setembro de 2018.

**Universidade Federal da Bahia, Escola de Medicina Veterinária e Zootecnia, Salvador, BA, BR.

***Universidade Federal da Bahia, Instituto de Ciências da Saúde, Salvador, BA, BR.

****Universidade Federal da Bahia, Colegiado de Biotecnologia, Vitória da Conquista, BA, BR.

Autor para correspondência: jeansilva_1@hotmail.com.
} 
A proteína total representa um grande número de componentes na circulação, compreendendo duas grandes frações; albumina e globulinas (Thomas, 2010). As globulinas são divididas nas frações alfa, beta e gama, sendo o fibrinogênio e a ceruloplasmina, glicoproteínas, produzidas pelo fígado e destacadas como importantes PFA (Murata et al., 2004). O aumento na concentração sérica das PFA está associado às elevações das concentrações das citocinas IL-1, IL-6 e TNFalfa, sintetizadas principalmente pelas células mononucleares e responsáveis pelo estímulo ao fígado, principal órgão produtor de PFA (Bode et al., 2012; Bastos et al., 2018).

Apesar da promessa de grande aplicabilidade no diagnóstico, prognóstico e monitoramento da saúde animal, o uso das PFA na rotina laboratorial depende do fornecimento de valores basais em diferentes condições fisiológicas para serem usadas como de normalidade ou mesmo de referência (Eckersall et al., 2000). O estudo do comportamento das PFA é fundamental para a compreensão das alterações ao longo do desenvolvimento dos animais, visto que em bezerros foi possível observar aumentos na concentração sérica de PFA nos primeiros dias de vida (Tóthová et al., 2015), assim como em cabritos (Ulutas et al., 2017).

Com este trabalho procurou-se estudar a cinética da proteína total, fibrinogênio e ceruloplasmina em ovinos da raça Santa Inês nos primeiros cinco meses de vida.

\section{Material e métodos}

\section{Animais e local do experimento}

Foram utilizados 22 cordeiros saudáveis oriundos de matrizes inseminadas artificialmente, da raça Santa Inês, criados semiextensivamente na Fazenda Experimental da Escola de Medicina Veterinária e Zootecnia da Universidade Federal da Bahia (EMVZ/UFBA), no município de São Gonçalo dos Campos, a $110 \mathrm{~km}$ de Salvador, Bahia (12 $25^{\circ}$ " 58' Sul, $38^{\circ} 58^{\prime \prime} 01^{\prime}$ Oeste), região de clima tropical, com altos índices pluviométricos.

\section{Manejo das matrizes e suas respectivas crias}

As matrizes e cordeiros eram alojados em galpão apropriado para criação de ovinos, com cobertura e aberturas laterais adequadas para a ventilação, evitando assim o estresse térmico. A dieta das matrizes e cordeiros era à base de concentrado, feno de Tifton e a água era fornecida ad libitum.

As matrizes passaram por protocolos reprodutivos, sincronização de cio e inseminação por via laparoscópica, o que permitiu concentrar o número de partos em um mesmo período, favorecendo o delineamento proposto. Durante a gestação, houve monitoramento diário, com fornecimento de dieta adequada. Em relação ao momento do parto, este era monitorado e quando necessário foram utilizadas manobras obstétricas.

Durante o dia, como protocolo da fazenda, os animais eram soltos para que pudessem socializar, ingerir gramíneas e melhorar o bem-estar animal. E somente no fim de cada dia eram realocados para o galpão. Os cordeiros lactentes permaneciam em baias específicas e alimentavam-se com colostro e leite de suas respectivas mães. Em casos de dificuldade na amamentação e baixo reflexo de sucção, eram estimulados e acompanhados rotineiramente.
Os cordeiros apresentaram média de 2,100 $\pm 0,245 \mathrm{~kg}$ ao nascimento e foram vermifugados com 30 dias de vida com ivermectina a 1\% (IVOMEC®; Merial Ltd., Iselin, NJ, USA). A vacinação para combate de botulismo e clostridiose (Poli-Star@; Vallée S.A., São Paulo, SP, Brasil) e desmame ocorreram por volta do terceiro mês de vida.

\section{Delineamento experimental}

As amostras de sangue foram colhidas em tubos à vácuos sem e com anticoagulante (EDTA-K3; Becton-Dickinson, São Paulo, Brasil), acondicionadas em gelo durante o transporte ao longo de onze momentos: imediatamente após o parto (T0), 12 (T1), 24 (T2), 48 horas (T3), sete (T4), 15 (T5), 30 (T6), 60 (T7), 90 (T8), 120 (T9) e 150 dias de vida (T10).

Cabe ressaltar que algumas fêmeas entraram em trabalho de parto bem antes da data prevista, dificultando a colheita de algumas amostras dos tempos iniciais. Além disso, a variação do número de amostras nos demais tempos ocorreu devido a diferentes intercorrências durante o experimento, fator de exclusão da análise, ficando o número total de amostras por tempo: T0=10, T1=12, T2=19 T3=22, T4=20, T5=19, T6=19, $\mathrm{T} 7=18, \mathrm{~T} 8=16, \mathrm{~T} 9=15$, е $\mathrm{T} 10=13$.

\section{Análise laboratorial}

Na primeira semana de vida dos animais (T0 a T4) as análises da proteína total e do fibrinogênio plasmáticos foram processadas na própria fazenda e do T5 ao T10 no Laboratório de Diagnóstico das Parasitoses dos Animais/LDPA do Hospital de Medicina Veterinária da UFBA, com refratômetro clínico, pela técnica de desnaturação pelo calor (Foster et al., 1959), respectivamente. Estes procedimentos foram realizados sempre no mesmo dia da colheita.

Os soros foram armazenados à temperatura de $-20^{\circ} \mathrm{C}$, para a determinação da concentração da ceruloplasmina que se baseou em sua atividade oxidásica (Ramos et al., 2018; Schosinsky et al., 1974). A leitura da absorbância foi realizada em espectrofotômetro, com comprimento de onda de $540 \mathrm{~nm}$ e os resultados foram expressos em UI/L.

\section{Análise estatística}

Os dados foram analisados estatisticamente usando o programa Statistical Package for the Social Sciences, versão 18, (SPSS, Inc., Chicago, IL, USA). Os dados foram testados quanto à distribuição de normalidade pelo teste Kolmogorov-Smirnov e, observou-se distribuição não paramétrica para a ceruloplasmina, fibrinogênio e proteína total. As variações das concentrações ao longo do tempo foram testadas pelo teste de Friedman e para as comparações múltiplas entre os tempos utilizou-se o teste de Wilcoxon, comparando-se as medianas dos diversos tempos antes e após a ingestão do colostro. O nível de significância adotado foi de $5 \%$.

\section{Resultados e discussão}

Na tabela 1 e figura 1 encontram-se os valores para a proteína total plasmática, onde se observa uma variação significativa nas concentrações ao longo do tempo (Teste de Friedman, $P=0,000$ ). Por meio das comparações múltiplas pelo teste de Wilcoxon foi possível verificar que o T0 diferiu dos demais tempos pós 
colostro ( $p<0,05)$. As medianas variaram de $5,1 \mathrm{~g} / \mathrm{dL}$ (T0) a $7,8 \mathrm{~g} /$ $\mathrm{dL}$ (T1), com valores mínimo e máximo de $4,8 \mathrm{~g} / \mathrm{dL}$ e $9,2 \mathrm{~g} / \mathrm{dL}$, para estes mesmos tempos, respectivamente.

David et al. (2012) trabalhando com cordeiros da raça Santa Inês, do nascimento até 15 dias de vida, obtiveram para a proteína total plasmática média de $5,83 \pm 0,65 \mathrm{~g} / \mathrm{dL}$ e de $5,48 \pm 0,59 \mathrm{~g} / \mathrm{dL}$ para animais de 1 a 2 meses. Neste mesmo intervalo de tempo, neste experimento os valores foram $6,8 \pm 0,9 \mathrm{~g} / \mathrm{dL}$ e $6,3 \pm 0,79 / \mathrm{dL}$, respectivamente. Por se tratar de animais da mesma raça e faixa etária, os manejos nutricional e sanitário devem ter influenciado nos valores obtidos.

Tabela 1: Valor mínimo, $1^{\circ}$ quartil, mediana, $3^{\circ}$ quartil e valor máximo da proteína total plasmática $(\mathrm{g} / \mathrm{dL})$ de ovinos Santa Inês do nascimento até o $5^{\circ}$ mês de vida.

\begin{tabular}{cccccccccccc}
\hline & T0 & T1 & T2 & T3 & T4 & T5 & T6 & T7 & T8 & T9 & T10 \\
& $(0 \mathrm{~h})$ & $(12 \mathrm{~h})$ & $(24 \mathrm{~h})$ & $(48 \mathrm{~h})$ & $(7 \mathrm{~d})$ & $(15 \mathrm{~d})$ & $(30 \mathrm{~d})$ & $(60 \mathrm{~d})$ & $(90 \mathrm{~d})$ & $(120 \mathrm{~d})$ & $(150 \mathrm{~d})$ \\
\hline Valor Mínimo & 4,8 & 6,6 & 6,2 & 5,6 & 5,4 & 5,7 & 5,9 & 5,1 & 5,0 & 5,0 & 5,0 \\
$1^{\circ}$ Quartil & 4,9 & 7,1 & 7,1 & 6,6 & 5,9 & 6,2 & 6,0 & 5,7 & 6,0 & 5,8 & 6,0 \\
Mediana & 5,1 & $7,8^{*}$ & $7,4^{*}$ & $7,1^{*}$ & $6,7^{*}$ & $6,4^{*}$ & $6,4^{*}$ & $6,1^{*}$ & $6,0^{*}$ & $5,9^{*}$ & $6,4^{*}$ \\
$3^{\circ}$ Quartil & 5,2 & 8,3 & 7,8 & 7,5 & 6,9 & 6,9 & 7,0 & 6,3 & 6,2 & 6,0 & 6,7 \\
Valor Máximo & 5,2 & 9,2 & 8,6 & 8,4 & 7,4 & 7,4 & 8,0 & 7,3 & 6,8 & 7,0 & 6,8 \\
Total & $\mathrm{n}=10$ & $\mathrm{n}=12$ & $\mathrm{n}=19$ & $\mathrm{n}=22$ & $\mathrm{n}=20$ & $\mathrm{n}=19$ & $\mathrm{n}=19$ & $\mathrm{n}=18$ & $\mathrm{n}=16$ & $\mathrm{n}=15$ & $\mathrm{n}=13$ \\
\hline
\end{tabular}

${ }^{*} \mathrm{P}<0,05$, significativo pelo teste de Wilcoxon quando comparado com T0; $h=$ horas, $d=$ dias

A ingestão do colostro promoveu um aumento na concentração da proteína total nas primeiras horas de vida (Figura 1). Os ovinos possuem placenta do tipo sindesmocorial e devido a anatomia das camadas que a compõe, a passagem de anticorpos entre a mãe e o feto é impossibilitada, o que justifica a dependência da ingestão de colostro para a aquisição de anticorpos (Souza et al., 2014). Além desse fator, a raça, idade e manejo alimentar podem influenciar nos valores da proteína total (Bezerra et al., 2009).

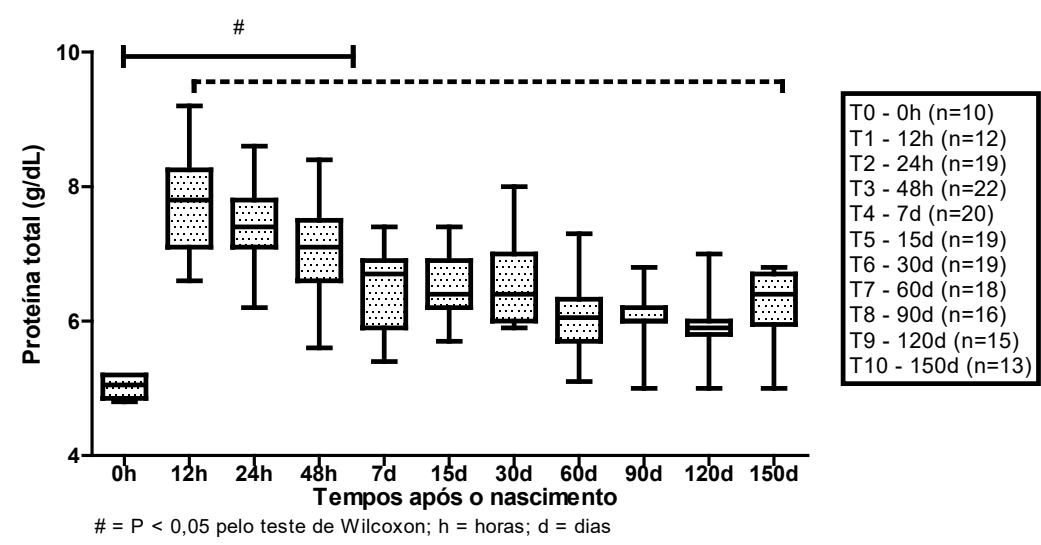

Figura 1: Distribuição das medianas de proteína plasmática total de ovinos Santa Inês do nascimento ao $5^{\circ}$ mês de vida.

O resultado das concentrações do fibrinogênio plasmático e sua distribuição encontram-se na tabela 2 e figura 2 , que não apresentou diferença estatística significante $(p<0,05)$ na análise dos diferentes tempos. Nesta pesquisa as medianas variaram de 150 a 400mg/dL nos diferentes tempos (Tabela 2).

Valores máximos fora do nível de normalidade foram observados em alguns animais do T2 (12h) ao T10 (150d), com pico de até $1.400 \mathrm{mg} / \mathrm{dL}$ no T3 (48h). O período inicial de vida é marcado por muitos desafios, como a adaptação extrauterina. Este processo de adaptação foi utilizado para justificar aumentos na concentração de PFA em bezerros e caprinos nos primeiros meses de vida (Tóthová et al., 2015; Ulutas et al., 2017). Por outro lado, o colostro pode exercer influência sobre a concentração sérica de PFA no período neonatal (Tóthová et al., 2015).

O efeito precoce de doenças não deve ser descartado, já que o status de higidez nem sempre reflete em concentrações baixas de PFA. Diferentes estímulos podem impactar no aumento da concentração destas proteínas, como o efeito do estresse, induzindo a produção de glicocorticoides, assim como o estímulo aos macrófagos. Estes eventos induzem a liberação de citocinas pró-inflamatórias promovendo estímulo a síntese de PFA pelo fígado (Murata et al., 2004).

Para a ceruloplasmina obteve-se medianas que variaram de 3,4 a $21,3 \mathrm{UI} / \mathrm{L}$, com valores máximo de 51,9UI/L e mínimo de 0,6 UI/L (Tabela 3 e figura 3). Constata-se que do total de amostras analisadas, 92\% estavam com valores abaixo de 30 UI/L, similares aos resultados obtidos por Matos (2005), que trabalhando com diferentes raças e manejo adequado obteve $92,5 \%$ abaixo deste valor, com medianas que variaram de 11,0 a $17,18 \mathrm{UI} / \mathrm{L}$.

Pelo teste de Friedman foi possível verificar a variação da ceruloplasmina ao longo do tempo $(P=0,001)$. Com base nas comparações múltiplas e analisando os resultados de T1 (12h) a T3 (48h) constata-se os valores mais baixos, muito embora às 24 horas (T2) tenha diferido estatisticamente $(p<0,05)$, em relação ao T0, com mediana de $8,1 \mathrm{UI} / \mathrm{L}$. Baixa concentração na primeira semana de vida e aumento com 14 e 28 dias de vida foram destacados em outro estudo, porém em caprinos (Ulutas et al., 2017). Fatores como a imaturidade hepática, contato com o ambiente nas primeiras semanas de vida e direcionamento das respostas imunológicas (Th1, Th2 e Th17) podem estar associados com as alterações observadas neste período (Chase, 2018; Kilpi, 2015).

A partir do sétimo dia (T4) as concentrações da ceruloplasmina aumentaram significativamente, atingindo pico nos tempos T8 (90 dias) e T9 (120 dias), com medianas de 21,3 e 20,6UI/L, respectivamente. Contudo, esses valores podem ser considerados dentro da normalidade, 
Tabela 2: Valor mínimo, $1^{\circ}$ quartil, mediana, $3^{\circ}$ quartil e valor máximo do fibrinogênio $(\mathrm{mg} / \mathrm{dL})$ de ovinos Santa Inês do nascimento até o $5^{\circ}$ mês de vida

\begin{tabular}{cccccccccccc}
\hline & $\begin{array}{c}\mathrm{T} 0 \\
(\mathrm{Oh})\end{array}$ & $\begin{array}{c}\mathrm{T} 1 \\
(12 \mathrm{~h})\end{array}$ & $\begin{array}{c}\mathrm{T} 2 \\
(24 \mathrm{~h})\end{array}$ & $\begin{array}{c}\mathrm{T} 3 \\
(48 \mathrm{~h})\end{array}$ & $\begin{array}{c}\mathrm{T} 4 \\
(7 \mathrm{~d})\end{array}$ & $\begin{array}{c}\mathrm{T} 5 \\
(15 \mathrm{~d})\end{array}$ & $\begin{array}{c}\mathrm{T} 6 \\
(30 \mathrm{~d})\end{array}$ & $\begin{array}{c}\mathrm{T} 7 \\
(60 \mathrm{~d})\end{array}$ & $\begin{array}{c}\mathrm{T} 8 \\
(90 \mathrm{~d})\end{array}$ & $\begin{array}{c}\mathrm{T} 9 \\
(120 \mathrm{~d})\end{array}$ & $\begin{array}{c}\mathrm{T} 10 \\
(150 \mathrm{~d})\end{array}$ \\
\hline Valor Mínimo & 100 & 100 & 100 & 100 & 100 & 100 & 100 & 100 & 200 & 100 & 100 \\
$1^{\circ}$ Quartil & 100 & 100 & 100 & 100 & 200 & 200 & 100 & 200 & 400 & 100 & 100 \\
Mediana & 150 & 200 & 100 & 200 & 300 & 300 & 200 & 300 & 400 & 200 & 200 \\
$3^{\circ}$ Quartil & 275 & 400 & 500 & 300 & 600 & 600 & 600 & 400 & 550 & 500 & 400 \\
Valor Máximo & 300 & 1200 & 900 & 1400 & 1000 & 1200 & 800 & 900 & 800 & 600 & 700 \\
Total & $\mathrm{n}=10$ & $\mathrm{n}=12$ & $\mathrm{n}=19$ & $\mathrm{n}=22$ & $\mathrm{n}=20$ & $\mathrm{n}=19$ & $\mathrm{n}=19$ & $\mathrm{n}=18$ & $\mathrm{n}=16$ & $\mathrm{n}=15$ & $\mathrm{n}=13$ \\
\hline
\end{tabular}

$\mathrm{h}=$ horas, $\mathrm{d}=$ dias

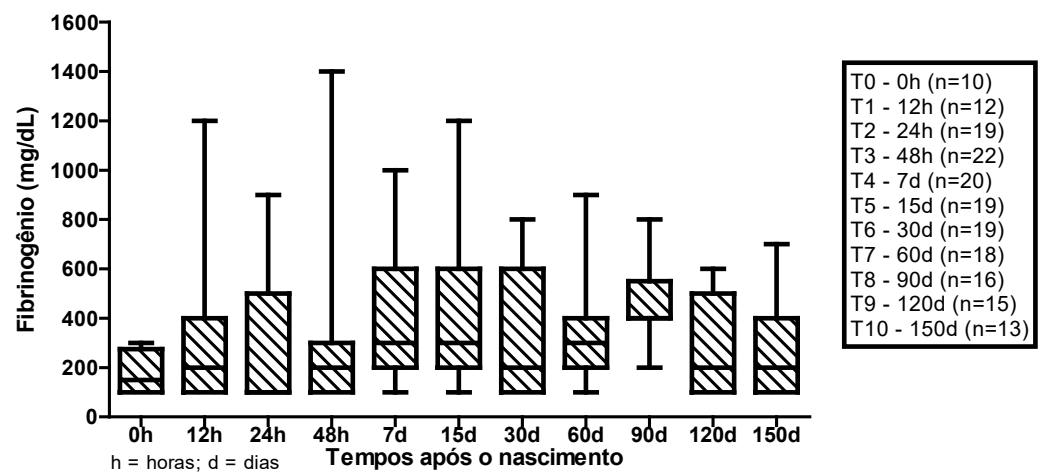

Figura 2: Distribuição das medianas do fibrinogênio de ovinos Santa Inês do nascimento ao $5^{\circ}$ mês de vida.

Tabela 3: Valor mínimo, $1^{\circ}$ quartil, mediana, $3^{\circ}$ quartil e valor máximo da ceruloplasmina $(\mathrm{UI} / \mathrm{L})$ de ovinos nascimento até o $5^{\circ}$ mês de vida

\begin{tabular}{cccccccccccc}
\hline & $\begin{array}{c}\mathrm{T} 0 \\
(\mathrm{Oh})\end{array}$ & $\begin{array}{c}\mathrm{T} 1 \\
(12 \mathrm{~h})\end{array}$ & $\begin{array}{c}\mathrm{T} 2 \\
(24 \mathrm{~h})\end{array}$ & $\begin{array}{c}\mathrm{T} 3 \\
(48 \mathrm{~h})\end{array}$ & $\begin{array}{c}\mathrm{T} 4 \\
(7 \mathrm{~d})\end{array}$ & $\begin{array}{c}\mathrm{T} 5 \\
(15 \mathrm{~d})\end{array}$ & $\begin{array}{c}\mathrm{T} 6 \\
(30 \mathrm{~d})\end{array}$ & $\begin{array}{c}\mathrm{T} 7 \\
(60 \mathrm{~d})\end{array}$ & $\begin{array}{c}\mathrm{T} 8 \\
(90 \mathrm{~d})\end{array}$ & $\begin{array}{c}\mathrm{T} 9 \\
(120 \mathrm{~d})\end{array}$ & $\begin{array}{c}\mathrm{T} 10 \\
(150 \mathrm{~d})\end{array}$ \\
\hline Valor Mínimo & 0,6 & 0,6 & 1,2 & 0,6 & 1,2 & 5,6 & 4,3 & 7,5 & 8,7 & 1,8 & 5 \\
10. Quartil $^{\circ}$ & 0,6 & 2,5 & 4,1 & 1,6 & 4,4 & 7,5 & 8,1 & 9,4 & 15,8 & 15,6 & 10,0 \\
Mediana & 3,4 & 5,0 & $8,1^{*}$ & 3,1 & $9,4^{*}$ & $13,8^{*}$ & $11,9^{*}$ & $12,5^{*}$ & $21,3^{*}$ & $20,6^{*}$ & $14,4^{*}$ \\
$3^{\circ}$. Quartil & 4,8 & 10,0 & 21,6 & 5,9 & 16,9 & 20,0 & 18,1 & 18,1 & 25,5 & 24,4 & 17,5 \\
Valor Máximo & 5,6 & 15,6 & 48,1 & 13,1 & 44,4 & 50,6 & 51,9 & 34,4 & 33,1 & 33,8 & 25,0 \\
\multicolumn{1}{c}{ Total } & $\mathrm{n}=10$ & $\mathrm{n}=12$ & $\mathrm{n}=19$ & $\mathrm{n}=22$ & $\mathrm{n}=20$ & $\mathrm{n}=19$ & $\mathrm{n}=19$ & $\mathrm{n}=18$ & $\mathrm{n}=16$ & $\mathrm{n}=15$ & $\mathrm{n}=13$ \\
\hline
\end{tabular}

${ }^{*} \mathrm{P}<0,05$, significativo pelo teste de Wilcoxon quando comparado com $\mathrm{T0} ; \mathrm{h}=$ horas, $\mathrm{d}=$ dias

segundo Matos (2005). Em condições fisiológicas e avaliando o comportamento de PFA nos primeiros 30 dias de vida em bezerros saudáveis, Tóthová et al. (2015) também encontraram aumento significativo da ceruloplasmina com 7 dias de vida. Os mesmos fatores justificados por estes autores podem ser citados aqui, tais como: adaptação extrauterina, estresse, infecção ou doenças como (Doença respiratória, diarreia e infecções umbilicais). Outro fator importante é o efeito da ingestão de colostro que pode estar associada aos aumentos na concentração sérica de PFA, no período neonatal.

O estado clínico de higidez em um rebanho pode não refletir a baixa concentração de PFA em um indivíduo. As PFA elevamse precocemente, fato este que justifica a sua utilização como biomarcador precoce de doenças (Eckersall e Bell, 2010). Além disso, é difícil a obtenção de testes de diagnóstico clínico $100 \%$ sensíveis e específicos. Como exemplo, em bezerros, alguns autores relatam sensibilidade de 5,9\% para o uso da auscultação na detecção de doença respiratória (Buczinski et al., 2014). A não detecção de um verdadeiro positivo, aquele que tem a doença e o teste de diagnóstico foi positivo, e a influência precoce de uma doença podem influenciar na concentração sérica obtida para a ceruloplasmina. Estes fatores podem justificar as alterações observadas no nosso estudo.

Embora a cinética de proteínas seja o escopo deste trabalho, analisando os valores máximos obtidos, observa-se que a ceruloplasmina, a exemplo do fibrinogênio, apresentou a partir do T3 resultados acima daqueles considerados de normalidade por Matos (2005). Muito provavelmente, o mesmo fator influenciou a elevação das concentrações dessa proteína, nos mesmos animais. Por outro lado, poucos trabalhos foram encontrados na literatura referindo valores de normalidade e a elevação da ceruloplasmina em ovinos enfermos. Costa et al. (2010) constataram também um aumento na concentração desta proteína, 108 horas após a inoculação de Staphylococcus aureus; isto é: de 27,57 $\pm 10,19 \mathrm{mg} /$ $\mathrm{dL}$ para $169,99 \pm 77,70 \mathrm{mg} / \mathrm{dL}$, praticamente oito vezes acima, mostrando a importância da obtenção de valores basais desta proteína para a espécie e sua relevância como marcador biológico de infecções bacterianas.

Para avaliação clínica, estudos de caso-controle avaliando os parâmetros de performance (Sensibilidade e especificidade) fornecerão um melhor entendimento da importância clínica das proteínas em questão. Poucos estudos abordam a ceruloplasmina como marcador biológico de doenças em cordeiros e a 


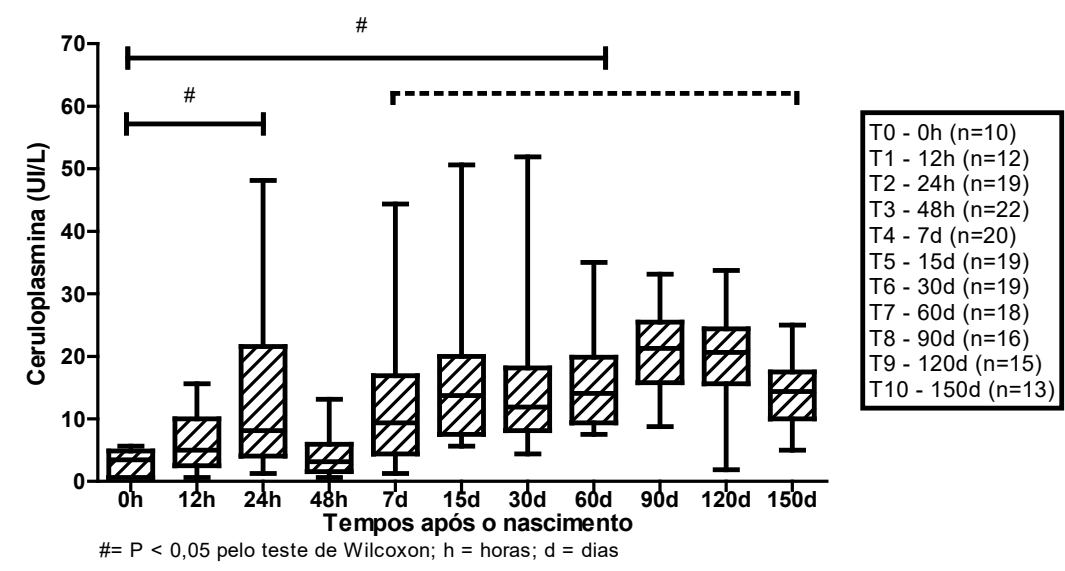

Figura 3: Distribuição das medianas da ceruloplasmina de ovinos do nascimento ao $5^{\circ}$ mês de vida. determinação da sensibilidade, especificidade e pontos de corte são fundamentais, não somente para pesquisas, mas também para a utilização em rotinas hospitalares.

\section{Conclusão}

De acordo com o protocolo experimental foi possível estabelecer a cinética das proteínas estudadas, observar os principais momentos de alterações na concentração sérica e os possíveis fatores associados. Este estudo contribui com uma abordagem detalhada do comportamento da proteína total, fibrinogênio e ceruloplasmina em cordeiros da raça Santa Inês e destaca a necessidade de estudos com foco na importância clínica.

\section{Referências}

BASTOS, B.L.; ROCHA-FILHO, J.T.R.; SANTANA, Í.P.; MEYER, R.; GUIMARÃES, J.E. Aplicabilidade da proteína haptoglobina como bioindicador de saúde na bovinocultura de corte e leite. PUBVET, 12, 130, 2018.

BEZERRA, L.R.; SILVA, A.M.A.; AZEVEDO, S.A.; RODRIGUES, O.G.; AZEVEDO, P.S.; MENDES, R.S. Concentrações séricas proteicas e minerais de cordeiros alimentados artificialmente com leite enriquecido com spirulinaplatentensis. Acta Veterinaria Brasílica, v.3, n.3, p.132-137, 2009.

BODE, J.G.; ALBRECHT, U.; HÄUSSINGER, D.; HEINRICH, P.C.; SCHAPER, F. Hepatic acute phase 384 proteins - Regulation by IL-6- and IL-1-type cytokines involving STAT3 and its crosstalk 385 with NF-kB-dependent signaling. European Journal of Cell Biology, v. 91, p.496-505, 2012.

BUCZINSKI, S.; FORTÉ, G.; FRANCOZ, D.; BÉLANGER, A.M. Comparison of thoracic auscultation, clinical score, and ultrasonography as indicators of bovine respiratory disease in preweaned dairy calves. Journal of veterinary internal medicine, 28(1), 234-242, 2014.

CHASE, C. Enteric Immunity Happy Gut, Healthy Animal. Veterinary Clinics Food Animal, v. 34, p. 1-18, 2018.

COSTA, N.A.; SIMÃO, L.C.V.; SANTOS, R.A.; AFONSO, J.A.B.; FAGLIARI, J.J.; CARDOSO, E.C.; SOARES, P.C.; MENDONÇA, C.L. Proteinograma e teores de cobre, ferro e zinco no soro sanguíneo de ovelhas da raça Santa Inês com mastite experimental por Staphylococcus aureus. Pesquisa Veterinária Brasileira, v. 30(5), p. 435-442, 2010.

DAVID, C.M.G.; LUQUETTI, B.C.; DA COSTA, R.L.D.; BONELLO, F.L. Padrão hematológico de cordeiros da raça Santa Inês criados sob manejo semi-extensivo na região oeste do estado de São Paulo. Boletim de Indústria Animal, N. Odessa, v.69, n.1, p.079084, 2012.

ECKERSALL, P.D. Recent advances and future prospects for the use of acute phase proteins as markers of disease in animals. Revue de Médecine Vétérinaire, v. 151, p. 577-584, 2000.

ECKERSALL, P.D.; BELL, R. Acute phase proteins: Biomarkers of infection and inflammation in veterinary medicine. Veterinary Journal, v. 185, n. 1, p. 23-27, 2010.
FOSTER, J.B.T.; NATALE, A.; DOTTI, L.B. Determination of plasma fibrinogen by needs of centrifugation after heating. American Journal of Clinical Pathology, v.31, n.1, p.42-5, 1959.

KILPI, A.J. Serum concentrations of globulins, albumin and serum amyloid a of neonatal lambs and associations with weight gain during summer rearing period. 2015. 38f. Doctoral teses Institute of Veterinary Medicine and Animal Sciences - Estonian University of Life Sciences, 2015.

MATOS, J.R. Proteínas de fase aguda em borregos e em ovelhas nos períodos de pré-parto e lactação. 2005. 56f. Dissertação - Escola de Medicina Veterinária e Zootecnia -Universidade Federal da Bahia, Salvador, 2005.

MURATA, H.; SHIMADA, N.; YOSHIOKA, M. Current research on acute phase proteins in veterinary diagnosis: An overview. Veterinary Journal, v. 168, n. 1, p. 28-40, 2004.

RAMOS, J.S.; KLUG, F.S.F.; BASTOS, B.L.; ROCHA FILHO, J.T.R.; AYRES, M.C.C.; GUIMARÃES, J.E. Determinação da concentração sérica de proteínas de fase aguda em equinos por técnicas espectrofotométricas. PUBVET, 12, 139, 2018.

SCHOSINSKY, K.H.; LEHMANN, H.P.; BEELER, M.F. Measurement of ceruloplasmin from its oxidase activity in serum by use of o-dianisidine dihydrochloride. Clinical Chemistry, v. 20, p. 1556-1563, 1974.

SOUZA, D.F.D.; MONTEIRO, A.L.G.; DITTRICH, R.L.; SCHMIDT, E.M.D.S.; FERNANDES, S.R.; BELTRAME, O.C. Dinâmica pré e pós-colostral de parâmetros bioquímicos em cordeiros. Ciência Animal Brasileira, 313-321, 2014.

THOMAS, J.S. Overview of plasma proteins. In: FELDMAN, B.F.; ZINKL, J.G.; JAIN, N.C. (Eds.). Schalm's veterinary hematology. Philadelphia: Lippincott Williams and Wilkins, 2010, p.891-898.

TÓTHOVÁ, C.; NAGY, O.; NAGYOVÁ, V.; KOVÁČ, G. Changes in the Concentrations of Acute Phase Proteins in Calves during the first Month of Life / Promene Koncentracije Proteina Akutne Faze Tokom Prvog Meseca Života Teladi. Acta Veterinaria, v. 65, n. 2, p. 260-270, 2015.

ULUTAS, P.A.; ULUTAS, B.; KIRAL, F.; ASICI, G. S. E.; GULTEKIN, M. Changes of acute phase protein levels in Saanen goat kids during neonatal period. Small Ruminant Research, v. 146, p. 33-36, 2017.

VIANA, J.G.A. Panorama Geral da Ovinocultura no Mundo e no Brasil. Revista Ovinos, Ano 4, N 12, Porto Alegre, março de 2008. 